\title{
A NEW METHOD TO IDENTIFY NEARBY, YOUNG, LOW-MASS STARS
}

\author{
David R. Rodriguez ${ }^{1}$, M. S. BesSell ${ }^{2}$, B. ZuCKerman ${ }^{1}$, AND JoEl H. KASTNER ${ }^{3}$ \\ ${ }^{1}$ Department of Physics \& Astronomy, University of California, Los Angeles, CA 90095, USA; drodrigu@astro.ucla.edu \\ 2 The Australian National University, Cotter Road, Weston Creek, ACT 2611, Australia \\ ${ }^{3}$ Center for Imaging Science, Rochester Institute of Technology, 54 Lomb Memorial Drive, Rochester, NY 14623, USA \\ Received 2010 September 2; accepted 2010 October 12; published 2011 January 4
}

\begin{abstract}
We describe a new method to identify young, late-type stars within $\sim 150 \mathrm{pc}$ of the Earth that employs visual or near-infrared (NIR) data and the GALEX GR4/5 database. For spectral types later than K5, we demonstrate that the ratio of GALEX near-ultraviolet to visual and NIR emission is larger for stars with ages between 10 and $100 \mathrm{Myr}$ than for older, main-sequence stars. A search in regions of the sky encompassing the TW Hya and Scorpius-Centaurus Associations has returned 54 high-quality candidates for follow up. Spectroscopic observations of 24 of these M1-M5 objects reveal Li $6708 \AA$ absorption in at least 17 systems. Because GALEX surveys have covered a significant fraction of the sky, this methodology should prove valuable for future young star studies.
\end{abstract}

Key words: open clusters and associations: individual (Lower Centaurus Crux, Upper Centaurus Lupus, TW Hydrae) - stars: evolution - stars: pre-main sequence - ultraviolet: stars

Online-only material: color figures

\section{INTRODUCTION}

The last two decades have seen the discovery of a number of moving groups and associations near the Earth containing young stars with ages in the range $\sim 10-100 \mathrm{Myr}$ (for reviews, see Zuckerman \& Song 2004 and Torres et al. 2008). By studying stars in these moving groups, we can observe the evolution of stellar properties as a function of age. Identifying additional members, particularly fainter, low-mass stars, is key to understanding the processes involved in the earliest stages of stellar, substellar, and massive planet evolution.

Low-mass stars with ages ranging from 10 to $100 \mathrm{Myr}$ are luminous sources of X-rays with respect to their bolometric luminosities (Kastner et al. 1997; Zuckerman \& Song 2004; Preibisch \& Feigelson 2005; Torres et al. 2006, and references therein). Several successful searches for nearby young stars exploited their characteristically large X-ray activities to identify promising candidates (Kastner et al. 1997; Song et al. 2003; Torres et al. 2006). In addition to X-ray activity, however, young, low mass stars also exhibit larger chromospheric activity than their older counterparts (as measured by the $R_{\mathrm{HK}}^{\prime}$ index; see Mamajek \& Hillenbrand 2008 and references therein). Higher chromospheric activity is correlated with increased ultraviolet emission in stars (Walkowicz \& Hawley 2009). Hence, when compared to the older population of low-mass stars, young stars will stand out as being UV-bright (e.g., see Figure 3 in Guinan \& Engle 2009).

The Galaxy Evolution Explorer satellite (GALEX; Martin et al. 2005) has performed a number of large area surveys in the far-ultraviolet (FUV; 1344-1786 ̊) and near-ultraviolet (NUV; 1771-2831 Å). The All-sky Imaging Survey (AIS), in particular, has covered $\sim 3 / 4$ of the sky, and hence represents an excellent tool to identify UV-bright sources. However, in order not to damage its detectors, GALEX generally does not point within $10^{\circ}$ of the galactic plane. Even with this limitation, GALEX has already explored the regions covered by many nearby, young stellar associations (Zuckerman \& Song 2004; Torres et al. 2008).

A previous study performed in the Taurus and Upper Scorpius (US) regions used GALEX to identify candidate young stars (Findeisen \& Hillenbrand 2010). However, these regions are populated by stars with ages $\leqslant 5$ Myr. We have successfully applied a similar strategy (described in Section 2) to search for nearby stars with ages 10-100 Myr and with spectral types later than K5 using archival data available in the GALEX GR4/5 data release.

\section{METHOD}

The search strategy we have employed consists of identifying UV-excess, late-type objects using NUV-Optical and NUV-NIR colors. With available proper motion data from the literature, we perform a $U V W$ space velocity analysis and the best candidate objects are selected for spectroscopic follow up. We describe our method in detail in Sections 2.1-2.3.

\subsection{GALEX (UV-based) Identification of Candidate Nearby, Young Stars}

To examine whether 10-100 Myr old stars stand out in the ultraviolet, we compare the absolute NUV magnitudes for stars in Torres et al. (2008), which list members of many of the young, nearby stellar associations, with the "Catalogue of Nearby Stars, 3rd Edition" by Gliese \& Jahreiß (1991); most of the latter are considerably older than a few 100 Myr. In particular, we used the catalog provided in Stauffer et al. (2010), which lists accurate coordinates for most of the stars in Gliese \& Jahreiß (1991). To identify UV sources we make use of CasJobs, ${ }^{4}$ an interface to query the GALEX database. The results are displayed in Figures 1 and 2. It is clear from these figures that young stars stand out from the older population. In particular, stars with $V-K \gtrsim 3.5(\sim \mathrm{M} 0)$ and ages as old as $70 \mathrm{Myr}$ (the $\mathrm{AB}$ Dor group in Torres et al. 2008) are brighter in NUV than the older Gliese stars; the only exceptions are flare stars (from Gershberg et al. 1999 or SIMBAD) and some multiple systems (from Dommanget \& Nys 2002 and Pourbaix et al. 2004). GALEX has a point-spread function (PSF) full width at half-maximum (FWHM) of 5" and, hence, cannot resolve binary systems with much smaller separations. Additionally, in some close binary systems (such as RS CVn-type systems) the component stars can interact with one another and may produce additional UV

\footnotetext{
4 http://mastweb.stsci.edu/gcasjobs/
} 


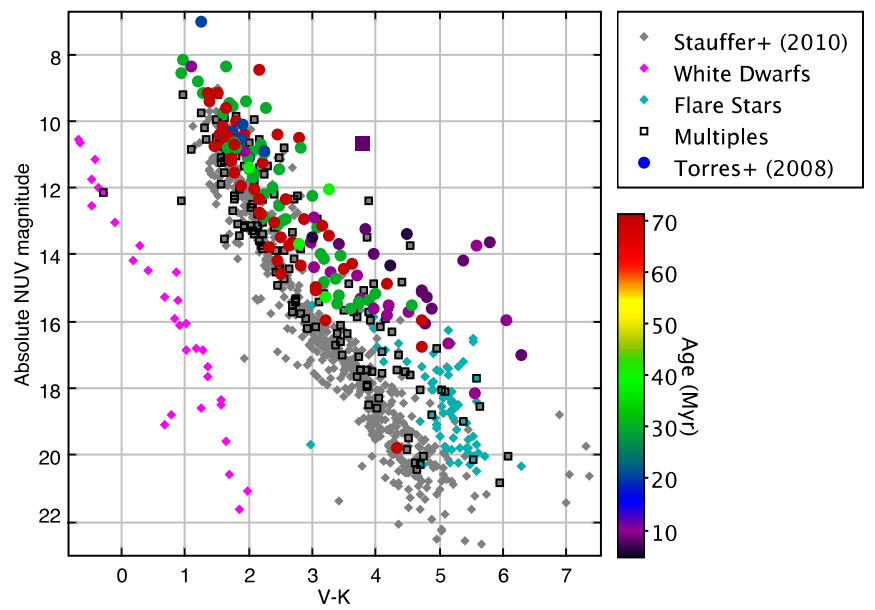

Figure 1. Circles, color-coded by age (grayscale in print version), are objects in Torres et al. (2008) that were matched in GALEX. TW Hya is displayed as a large square. Putative AB Dor member BD +012447 is located at $V-K \sim 3$ and absolute NUV magnitude $\sim 20$. All other symbols are stars from Stauffer et al. (2010), which provides stars in the Gliese catalog (Gliese \& Jahreiß 1991). Different symbols highlight some of the known multiple, white dwarfs, and flare star systems. The Gliese stars left of the main sequence are likely to have incorrect distances, $V$-band magnitudes, or both (some such systems were corrected when updated information was available). In general, the Gliese low-mass stars (K5/K7 and later, $V-K \geqslant 3)$ are older and fainter in NUV than stars in young associations by 2 mag on average, the notable exception being flare stars and some multiples. Multiples among the Torres et al. (2008) stars are not labeled.

(A color version of this figure is available in the online journal.)

emission. The only exception to this trend of brighter NUV magnitudes for young stars is BD +01 2447, classified by Torres et al. (2008; see also references therein) as an AB Dor member, with $V-K \sim 4.3$, which we discuss later in Section 3.4. Figure 2, which displays NUV- $V$ colors, also shows how young stars stand out. Reddening toward these objects can significantly affect the shorter wavelengths. Findeisen \& Hillenbrand (2010) apply a reddening curve in which $A_{\mathrm{NUV}} / A_{V}=2.63$; however, using the reddening curve of Cardelli et al. (1989) and $R_{V}=3.1$, we obtain $A_{\mathrm{NUV}} / A_{V}=2.96$. While most Gliese stars are within $25 \mathrm{pc}$, most of the young stars are more distant. Regardless of the exact value for $A_{\mathrm{NUV}} / A_{V}$, extinction toward the younger, more distant objects will generally be higher than for the nearby Gliese stars. Thus, the effect of extinction will be to bring the locus of the young star populations closer to the Gliese old star locus. That is, the intrinsic separation in absolute NUV magnitude and in NUV $-V$ between young and old stars is, if anything, somewhat greater on average than indicated in Figures 1 and 2.

Young, early-M stars $(V-K \sim 3.5)$ in these associations (with ages 10-30 Myr) tend to have absolute NUV magnitudes between 15 and 16 (see Figure 1). The $3 \sigma$ sensitivity for the GALEX AIS survey is $22.0 \mathrm{mag}$ for each 100 -s exposure, which means that these young stars could be detected at the $3 \sigma$ level out to at least 160 pc. Findeisen \& Hillenbrand (2010) estimate that GALEX can detect unextincted photospheres of K5 stars out to 140 pc with 300-s exposures, implying absolute NUV magnitudes of about 17. Mid-K stars with ages of $70 \mathrm{Myr}$, however, have absolute NUV magnitudes generally brighter than 15 , suggesting that even at $70 \mathrm{Myr}$, these stars will be about 2 mag brighter than their older counterparts (as illustrated in Figure 1 at $V-K \sim 3$ ). As previously mentioned, extinction toward these objects will cause them to have redder NUV-V colors - similar to that of older field stars. Extinction of (at least) $A_{V} \sim 0.7 \mathrm{mag}$ would be required for $70 \mathrm{Myr}$ old mid-K

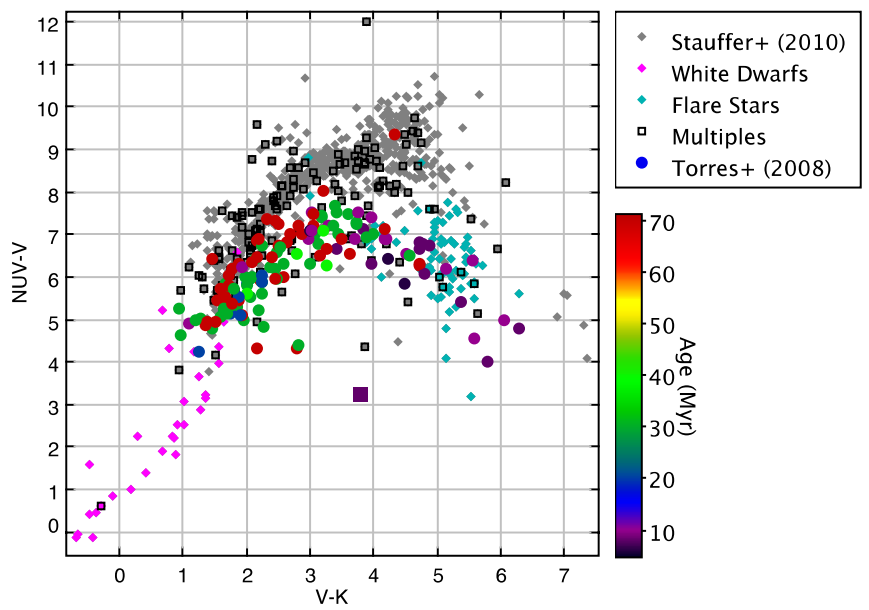

Figure 2. When plotting the members of young associations and the Gliese stars (see Figure 1) in NUV- $V$ color, the distinction between old and young, late-type $(\mathrm{K} 5 / \mathrm{K} 7$ and later, $V-K \geqslant 3)$ stars becomes more readily apparent. Objects with lower NUV- $V$ are brighter in NUV and generally younger. TW Hya is displayed as a large square. Putative AB Dor member BD +01 2447 is located at $V-K \sim 4.3$ and NUV-V $\sim 9.4$. We comment on both these systems in Section 3.4.

(A color version of this figure is available in the online journal.)

stars to have NUV magnitudes comparable to equally distant, but older, mid-K stars.

To see if these 10-100 Myr old systems also stand out as UV-bright in GALEX/Two Micron All Sky Survey (2MASS) colors, and to carry out a pilot study for the feasibility of a largescale program, we examine a region of sky encompassing the TW Hya Association (TWA). After defining a suitable search region (see Table 1), we select all objects detected in NUV (our initial search of the TWA also required detection in FUV). These sources are then cross-matched with the 2MASS Point Source Catalog (Cutri et al. 2003) with a matching radius of $2^{\prime \prime}$, somewhat larger than the GALEX pointing uncertainty $\left(\sim 1^{\prime \prime}\right.$; Morrissey et al. 2007). This allows us to produce color-color plots like the one in Figure 3. In these plots, galaxies tend to lie in a well-defined region of space (between about $0.5<J-K<2$ and $2<$ NUV $-J<7$ ) while stars follow a diagonal line from $J-$ $K \sim 0$ and NUV $-J \sim 2$ to $J-K \sim 1$ and NUV- $J \sim 13$. The black symbols in Figure 3 are the TWA stars from Torres et al. (2008), all of which lie below the stellar main sequence; TW Hya is the object with NUV $-J \sim 6$. The stellar locus is well defined by an average of the relations in Findeisen \& Hillenbrand (2010, see their Equation (2)) and we have plotted this as the thin solid line in Figure 4. Also shown are the 10-100 Myr old stars, which can again be distinguished from older field stars in that they lie below the stellar main sequence (i.e., they have UV excesses). The thick solid line represents the best linear fit to the locus of young stars, and illustrates the difference between the young and old stellar populations, particularly for the lower mass stars $(J-K \geqslant 0.7)$. While the separation is not as pronounced as in the NUV- $V$ plots, clearly these diagrams should enable one to select candidate young, low-mass stars using only GALEX and 2MASS colors.

In Figures 3 and 4, the young stars of the TWA all lie below the stellar sequence. However, there is some scatter, and not every object with UV excess will be a young star. For example, flare stars are known contaminants in young star X-ray searches, and the same is true for our UV-selection methods (see also Figures 1 and 2). We select objects with UV excesses similar 


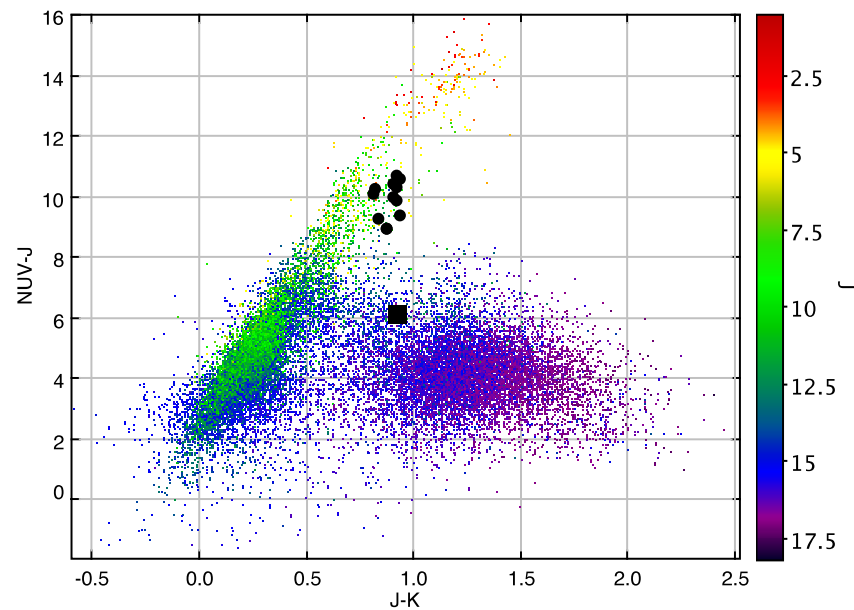

Figure 3. Color-color plot for the TWA search. The dots (color/grayscale based on $J$ magnitude) are the $G A L E X / 2$ MASS sources, while the larger black dots are known TWA members from Torres et al. (2008). The TWA member labeled as a large square is TW Hya itself. The cloud of sources between about $0.5<$ $J-K<2$ and $2<$ NUV- $J<7$ are generally distant galaxies and most can be removed from this plot by imposing a cut of $J \leqslant 14$. All TWA objects lie somewhat below the line of stellar sources (i.e., they have UV excesses; see Figure 4).

(A color version of this figure is available in the online journal.)

to those of the known young stars (defined algebraically by NUV $-J \leqslant 10.20(J-K)+2.2)$ and $J-K$ between 0.7 and 1 , as most M-type stars have $J-K$ colors of about 0.8 . A cut of $J \leqslant$ $14(J \leqslant 12$ for our initial TWA search) is imposed to eliminate faint, UV-bright galaxies (see Figure 3). Our selection criteria are illustrated in Figure 5 along with our candidate objects (see Section 3) and fits to the old and young stellar locus as described above (see also Figure 4).

\subsection{Proper Motions and UVW Space Velocities}

To distinguish between candidate young stars and other UV excess sources, we make use of proper motion information available in the literature. In particular, we use the USNOB1 (Monet et al. 2003), UCAC3 (Zacharias et al. 2010), and PPMX (Röser et al. 2008) proper motion catalogs, which can be accessed from the TOPCAT ${ }^{5}$ Virtual Observatory tool. In some cases, we also check the PPMXL catalog (Roeser et al. 2010). Our UV excess sources are matched against all three catalogs, compared with proper motions of members in the association of interest, and then merged while removing duplicate entries. In cases where one catalog has a proper motion that is discrepant from the others, we favor the proper motions listed in UCAC3 and PPMX.

We next estimate galactic space velocities $(U V W)$ for candidate stars. This step requires estimates for distances and radial velocities. In order to obtain photometric distances, we estimate spectral types using available broadband photometry (for example, data from DENIS (The DENIS Consortium 2005) or the proper motion catalogs listed above). From the spectral type we estimate photometric distances using empirical and theoretical isochrones for young stars of ages 10 and 100 Myr (Song et al. 2003; Zuckerman \& Song 2004, and references therein). These distance estimates have uncertainties of $\sim 20 \%$. Because nearly all candidates lack radial velocities, we use these distance estimates (along with the positions and proper motions) to estimate

\footnotetext{
5 Available at http://www.starlink.ac.uk/topcat/
}

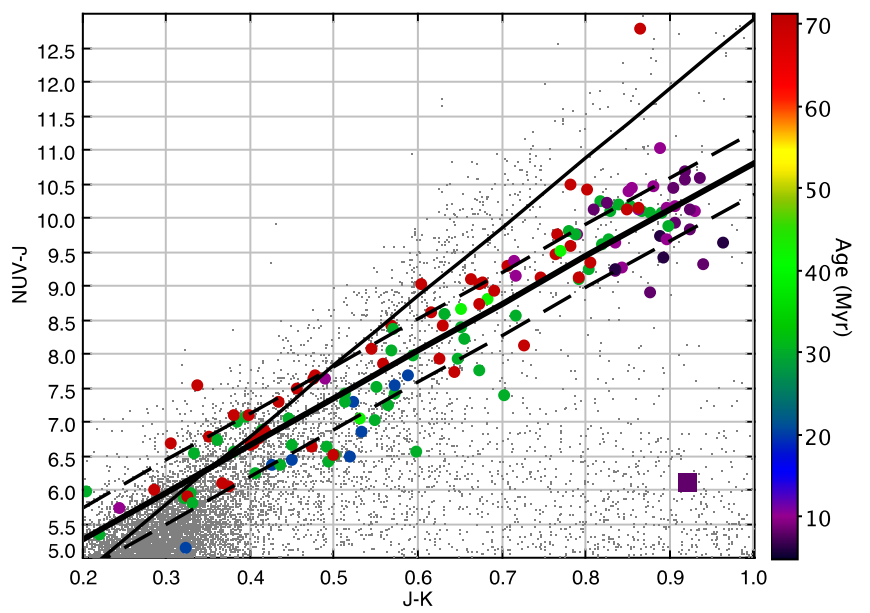

Figure 4. Small dots are the GALEX/2MASS sources in the vicinity of TWA (see the first line entry to Table 1); those near the bottom right are the upper portion of the galaxy distribution (see Figure 3). The circles are for stars in Torres et al. (2008), where the color/grayscale corresponds to the age of the association or moving group. TW Hya is the large purple square. The thin solid line represents the relations in Findeisen \& Hillenbrand (2010) and defines the stellar sequence as extrapolated from early-type stars. The thick solid line is the best-fit line to the young star sample and demonstrates (as in Figures 1 and 2) how young late-type stars stand out. Dashed lines outline the $1 \sigma$ variation among the young stars.

(A color version of this figure is available in the online journal.)

Galactic $U V W$ with respect to the Sun for a range of velocities extending from -80 to $80 \mathrm{~km} \mathrm{~s}^{-1}$. Zuckerman \& Song (2004) define a "good $U V W$ box" that contains nearly all young stars. If any object has $U V W$ within this box, that is, $U V W$ within 0 to $-15,-10$ to -34 , and +3 to $-20 \mathrm{~km} \mathrm{~s}^{-1}$, they are flagged for follow up. Listed in the next last column in Table 2 (see also table footnote) are the range of radial velocities for our candidate objects that give UVW in this box. In this case, $U$ is defined as positive toward the Galactic center.

Follow up for all candidates so identified involves first checking against known association members. In the TWA, for example, nearly all known members are recovered via the above methodology, including some that are not included in Torres et al. (2008) but are present in older compilations, such as those in Zuckerman \& Song (2004). The candidates are searched in SIMBAD as well, though most have no SIMBAD entry and only limited information in VizieR.

\subsection{Lithium Line Spectroscopy}

Candidates considered for spectroscopic follow up are those with a broad range of radial velocities that would give $U V W$ velocities consistent with nearby, young star status and photometric distances consistent with other members in the association of interest. The presence and strength of the Li $6708 \AA$ absorption in the spectrum can indicate whether or not the object is young (see Figure 3 in Zuckerman \& Song 2004 and the associated discussion in Section 5.1 of the same paper). A high-resolution spectrum can also be used to determine the radial velocity of the candidate object, which can then be used to better constrain its $U V W$ velocities.

Although the presence of Li for stars of the spectral types of interest (K5 and later) is a strong indication of youth, lithium burning is very sensitive to stellar mass (and thus spectral type). For example, $\beta$ Pic Moving Group stars ( 12 Myr) of spectral types M3-4 show very little Li in their spectra (Song et al. 2002; 


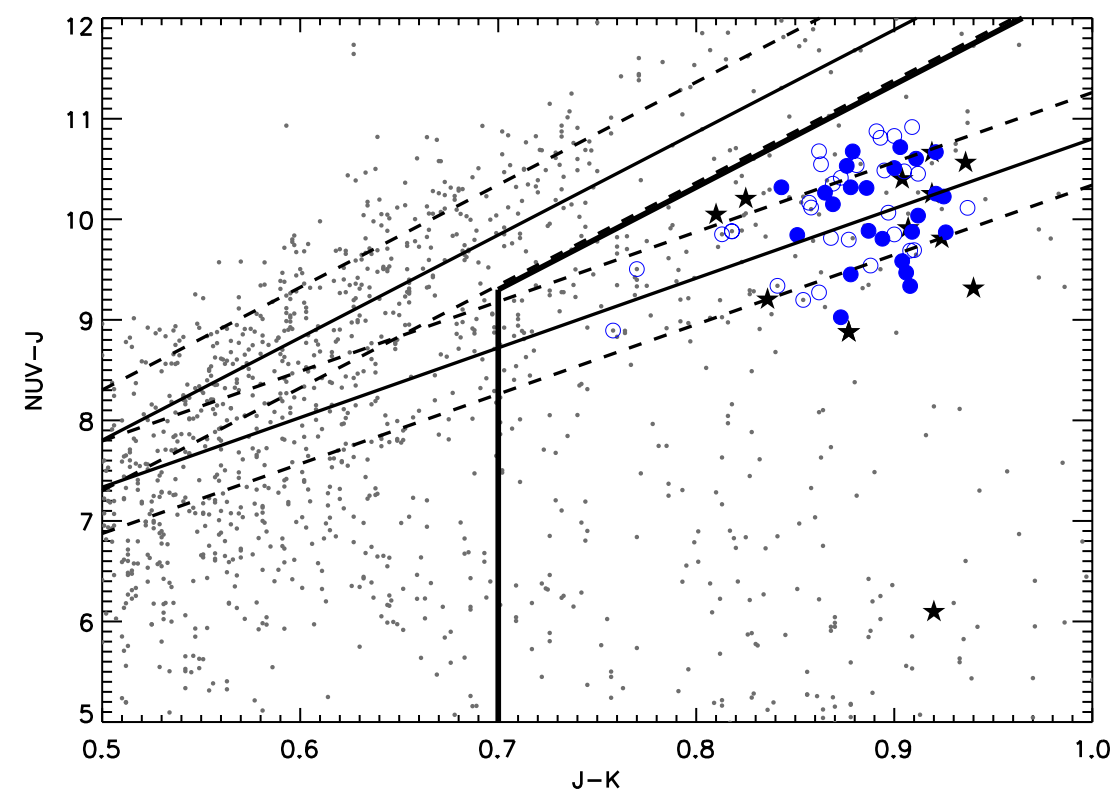

Figure 5. Our 54 UV-excess candidate stars are plotted as circles with filled circles denoting our spectroscopic sample. TWA members known previous to our study (namely, those listed in Torres et al. 2008) are plotted with star symbols. Our selection criteria are denoted by the thick black lines; the thin solid lines denote the relation in Findeisen \& Hillenbrand (2010) for the stellar sequence and our best-fit line to the young stars (see Figure 4). Both have their associated $1 \sigma$ errors as dashed lines. The gray dots are the GALEX/2MASS sources in the vicinity of TWA with $J \leqslant 14$, which removes many of the galaxies. While we select all objects that satisfy our color-color criteria, most do not have proper motions, distances, or $U V W$ consistent with those of young stars (see Sections 2.2 and 3.1).

(A color version of this figure is available in the online journal.)

Zuckerman \& Song 2004; Yee \& Jensen 2010). Lithium can be depleted more quickly than models predict resulting in older ages for a given lithium abundance (Yee \& Jensen 2010). Hence, a non-detection of lithium absorption will not immediately imply the system is old, particularly for mid-M spectral types. Because many of our candidates fall in this spectral range, a nondetection would imply a system is older than about $10 \mathrm{Myr}$, but does not provide an upper limit to this age. Such a system could, however, still be a member of a young association. In such cases, membership could be confirmed via accurate distance, proper motion, and radial velocity measurements.

\section{APPLICATION: IDENTIFICATION OF NEARBY YOUNG STARS IN AND NEAR THE TW Hya ASSOCIATION}

\subsection{NUV/NIR-based Candidate Identification}

We have used the method described in Section 2 to perform a search for young stars in the TWA $(\sim 8 \mathrm{Myr}, 40-60 \mathrm{pc}$; Zuckerman \& Song 2004; Torres et al. 2008, and references therein) and the Scorpius-Centaurus region (Preibisch \& Mamajek 2008). The Sco-Cen region is divided into a number of associations: US ( $\sim 5-6 \mathrm{Myr}$ old), Upper Centaurus-Lupus (UCL; $\sim 14-15 \mathrm{Myr}$ ), and Lower Centaurus-Crux (LCC; 11-12 Myr), which are located about $120-140$ pc distant (Preibisch \& Mamajek 2008). ${ }^{6}$ In order to limit our searches to a manageable number of objects (the GALEX catalog CasJobs search interface has built-in memory limits), we divided the Sco-Cen search into a set of four R.A./decl. centroids, each with a given radius (see Table 1 and Figure 6). There is some overlap between these regions, so the resulting lists of objects (after cross-correlating with 2MASS) were merged and duplicate entries were removed.

\footnotetext{
6 Based on Li abundances in late-type members of LCC and UCL, Song et al.
} (2010, unpublished) suggest that these regions are only $\sim 10$ Myr old.
Table 1

GALEX Searches

\begin{tabular}{lcccc}
\hline \hline Region $^{\mathrm{a}}$ & R.A. & Decl. & Radius & $\begin{array}{c}\text { GALEX/2MASS } \\
\text { Sources }\end{array}$ \\
\hline TWA & $11^{\mathrm{h}} 30^{\mathrm{m}}$ & $-35^{\circ}$ & $18^{\circ}$ & $47,101^{\mathrm{b}}$ \\
LCC 1 & $10^{\mathrm{h}} 56^{\mathrm{m}}$ & $-63^{\circ}$ & $15^{\circ}$ & 381,385 \\
LCC 2 & $12^{\mathrm{h}} 50^{\mathrm{m}}$ & $-60^{\circ}$ & $18^{\circ}$ & \\
UCL 1 & $14^{\mathrm{h}} 45^{\mathrm{m}}$ & $-45^{\circ}$ & $18^{\circ}$ & 548,324 \\
UCL 2 & $16^{\mathrm{h}} 20^{\mathrm{m}}$ & $-33^{\circ}$ & $18^{\circ}$ & \\
\hline
\end{tabular}

Notes. List of all searches performed. See also Figure 6. Number of stars quoted for LCC 1 region also includes stars in LCC 2; similarly for UCL 1 and UCL 2. a TWA: TW Hydra Association; LCC: Lower Centaurus-Crux; UCL: Upper Centaurus-Lupus.

${ }^{\mathrm{b}}$ Only the TWA search required detection in both NUV and FUV.

Candidate objects were selected via the technique described in Sections 2.1 and 2.2.

We identified $\sim 150$ promising candidates through this methodology. In order to select the best objects to observe, we selected only those with a broad range of possible radial velocities that would give $U V W$ consistent with those of young stars, and those with distances comparable to that of the TWA or Sco-Cen regions. After these cuts we had selected 10 TWA candidates and 44 Sco-Cen candidates. These 54 candidates are listed in Table 2, with spectral types estimated from photometric colors. These candidate objects are also displayed in Figure 5 along with known TWA members for comparison. Note, however, that the LCC and TWA are very close in the sky and have similar ages, so accurate distances are needed to determine in which association these targets belong. The TWA and Sco-Cen search fields are displayed in Figure 6, with candidate objects shown as open circles and those for which we obtained spectra (Table 3) shown as filled circles. 
Table 2

UV Selected Objects

\begin{tabular}{|c|c|c|c|c|c|c|c|c|c|c|}
\hline $\begin{array}{l}\text { R.A. } \\
\text { (deg) }\end{array}$ & $\begin{array}{l}\text { Decl. } \\
\text { (deg) }\end{array}$ & $\begin{array}{c}\text { 2MASS } \\
\text { Designation } \\
\end{array}$ & $\begin{array}{c}\mu_{\alpha} \\
\left(\operatorname{mas~yr}^{-1}\right)\end{array}$ & $\begin{array}{c}\mu_{\delta} \\
\left(\operatorname{mas~yr}^{-1}\right)\end{array}$ & $\begin{array}{l}J-K \\
(\mathrm{mag}) \\
\end{array}$ & $\begin{array}{c}\mathrm{NUV}-J \\
(\mathrm{mag})\end{array}$ & $\begin{array}{c}\text { Spectral } \\
\text { Type } \\
\end{array}$ & $\begin{array}{c}D \\
(\mathrm{pc})\end{array}$ & $\begin{array}{c}\text { RV Range } \\
\left(\mathrm{km} \mathrm{s}^{-1}\right)\end{array}$ & $\log L_{X} / L_{\mathrm{bol}}$ \\
\hline 156.337 & -42.6983 & $10252092-4241539$ & -47 & -2 & 0.91 & 10.04 & M2-M2.5 & 91 & $10: 30$ & -3.03 \\
\hline 156.509 & -41.0983 & $10260210-4105537$ & -46 & -3 & 0.9 & 9.58 & M3-M3.5 & 67 & $8: 31$ & -3.24 \\
\hline 165.8988 & -30.4137 & $11033571-3024494$ & -36 & -18 & 0.84 & 9.34 & M0 & 126 & $0: 26$ & $<-3.57$ \\
\hline 171.448 & -44.1741 & $11254754-4410267$ & -37 & -18 & 0.86 & 10.26 & M4-M4.5 & 75 & $2: 27$ & -2.33 \\
\hline 172.812 & -48.4411 & $11311482-4826279$ & -40 & -6 & 0.87 & 9.03 & M3-M3.5 & 130 & $17: 26$ & $<-3.24$ \\
\hline 174.079424 & -52.515057 & $11361906-5230542$ & -31 & -10 & 0.86 & 10.12 & M3-M3.5 & 123 & $4: 29$ & -2.80 \\
\hline 176.596286 & -52.647766 & $11462310-5238519$ & -46 & -14 & 0.89 & 9.88 & M4.5-M5 & 85 & $3: 28$ & $<-2.97$ \\
\hline 179.866105 & -45.172005 & $11592786-4510192$ & -44 & -24 & 0.87 & 10.15 & M4.5-M5 & 55 & $5: 21$ & -2.99 \\
\hline 179.99 & -26.3761 & $11595770-2622340$ & -32 & -17 & 0.85 & 9.20 & M3-M3.5 & 113 & $-2: 23$ & $<-3.35$ \\
\hline 181.698 & -19.3481 & $12064743-1920531^{\mathrm{a}}$ & -56 & -7 & 0.77 & 9.50 & K7-M0 & 71 & $2: 11$ & $<-3.57$ \\
\hline 182.1542 & -21.45811 & $12083700-2127291^{\mathrm{a}}$ & -73 & -36 & 0.81 & 9.85 & M3 & 60 & $-4: 21$ & $<-2.92$ \\
\hline 184.598 & -35.2527 & $12182363-3515098$ & -28 & -13 & 0.82 & 9.88 & M0.5-M1 & 101 & $2: 22$ & -3.19 \\
\hline 184.973163 & -74.33593 & $12195355-7420093$ & -34 & -14 & 0.89 & 10.81 & M3.5-M4 & 104 & $5: 28$ & -3.03 \\
\hline 186.714 & -33.2701 & $12265135-3316124$ & -62 & -25 & 0.91 & 9.34 & M5 & 65 & $-2: 23$ & $<-3.10$ \\
\hline 186.855 & -45.6685 & $12272529-4540065$ & -30 & -14 & 0.76 & 8.89 & K7-M0 & 149 & $2: 25$ & -3.11 \\
\hline 187.521722 & -44.04332 & $12300521-4402359$ & -44 & -18 & 0.88 & 10.53 & M4.5-M5 & 69 & $2: 24$ & $<-3.25$ \\
\hline 190.225488 & -45.27367 & $12405411-4516252$ & -35 & -13 & 0.91 & 10.92 & M3-M3.5 & 124 & $2: 26$ & $<-3.19$ \\
\hline 192.215415 & -45.939037 & $12485169-4556205$ & -27 & -14 & 0.9 & 10.07 & M4.5-M5 & 121 & $0: 23$ & $<-2.76$ \\
\hline 192.698969 & -42.53022 & $12504775-4231487$ & -34 & -18 & 0.82 & 9.88 & M4.5-M5 & 120 & $-1: 26$ & $<-2.76$ \\
\hline 194.322195 & -46.447998 & $12571732-4626527$ & -31 & -20 & 0.87 & 10.35 & M3.5-M4 & 146 & $2: 23$ & $<-2.95$ \\
\hline 196.442675 & -44.293636 & $13054624-4417370$ & -35 & -16 & 0.89 & 10.49 & M3-M3.5 & 143 & $6: 22$ & -2.78 \\
\hline 196.632647 & -44.919258 & 13063183-4455093 & -29 & -13 & 0.87 & 10.41 & M2-M2.5 & 138 & $0: 26$ & $<-3.28$ \\
\hline 197.870938 & -42.878288 & $13112902-4252418$ & -35 & -19 & 0.9 & 10.51 & M2-M2.5 & 121 & $0: 25$ & -2.93 \\
\hline 200.17506 & -46.976494 & $13204201-4658353$ & -29 & -20 & 0.91 & 9.69 & M4-M4.5 & 126 & $-3: 21$ & $<-2.92$ \\
\hline 201.775952 & -43.506329 & $13270622-4330227$ & -35 & -17 & 0.86 & 10.16 & M4.5-M5 & 118 & $0: 24$ & $<-2.80$ \\
\hline 202.060031 & -39.487438 & $13281440-3929147$ & -28 & -32 & 0.91 & 10.60 & M4-M4.5 & 84 & $-2: 11$ & $<-3.22$ \\
\hline 202.126198 & -39.568535 & $13283028-3934067$ & -32 & -14 & 0.91 & 9.69 & M4-M4.5 & 134 & $0: 21$ & $<-2.86$ \\
\hline 202.135325 & -42.695457 & $13283247-4241436$ & -44 & -36 & 0.93 & 10.23 & M3-M3.5 & 111 & $3: 18$ & $<-3.27$ \\
\hline 203.373612 & -37.88876 & $13332966-3753195$ & -23 & -20 & 0.91 & 10.45 & M4-M4.5 & 143 & $-7: 16$ & $<-2.89$ \\
\hline 203.880003 & -42.696892 & $13353120-4241488$ & -26 & -23 & 0.94 & 10.11 & M4-M4.5 & 123 & $-5: 17$ & $<-3.02$ \\
\hline 204.409991 & -47.608257 & $13373839-4736297$ & -32 & -23 & 0.91 & 9.47 & M4.5-M5 & 126 & $0: 23$ & $<-2.70$ \\
\hline 207.22411 & -48.84594 & $13485378-4850453$ & -33 & -18 & 0.86 & 10.68 & M3.5-M4 & 146 & $4: 22$ & $<-3.02$ \\
\hline 207.940297 & -37.700115 & $13514567-3742004$ & -26 & -36 & 0.92 & 10.25 & M2-M2.5 & 119 & $-9: 13$ & $<-3.43$ \\
\hline 208.190309 & -49.642429 & $13524567-4938327$ & -37 & -17 & 0.84 & 10.32 & M4.5-M5 & 112 & $1: 22$ & $<-2.75$ \\
\hline 210.906607 & -50.179924 & $14033758-5010477$ & -33 & -18 & 0.86 & 10.55 & M4-M4.5 & 98 & $-3: 17$ & $<-3.16$ \\
\hline 211.712131 & -50.98185 & $14065091-5058546$ & -28 & -24 & 0.9 & 10.83 & M4-M4.5 & 121 & $-3: 18$ & $<-3.04$ \\
\hline 216.715646 & -33.826576 & $14265175-3349356$ & -28 & -32 & 0.9 & 10.72 & M3.5-M4 & 139 & $-5: 14$ & $<-3.05$ \\
\hline 218.440042 & -31.41147 & $14334561-3124412$ & -20 & -30 & 0.89 & 9.54 & M4.5-M5 & 97 & $-8: 5$ & $<-2.88$ \\
\hline 219.305101 & -34.155811 & $14371322-3409209$ & -32 & -31 & 0.89 & 9.81 & M3-M3.5 & 145 & $-3: 15$ & $<-3.17$ \\
\hline 222.245008 & -32.402317 & $14485880-3224083$ & -34 & -32 & 0.8 & 9.84 & M4.5-M5 & 132 & $-4: 14$ & -2.38 \\
\hline 222.639972 & -34.341827 & $14503359-3420305$ & -29 & -31 & 0.88 & 9.45 & M4-M4.5 & 142 & $-11: 7$ & $<-2.90$ \\
\hline 226.646079 & -36.658264 & $15063505-3639297$ & -34 & -25 & 0.93 & 9.87 & M5-M5.5 & 118 & $-4: 13$ & $<-2.58$ \\
\hline 227.068891 & -34.579636 & $15081653-3434466$ & -31 & -32 & 0.91 & 9.87 & M4-M4.5 & 140 & $-5: 12$ & $<-2.86$ \\
\hline 228.050838 & -25.952211 & $15121220-2557079$ & -20 & -33 & 0.89 & 10.88 & $\mathrm{M} 2-\mathrm{M} 2.5$ & 99 & $-14: 3$ & $<-3.58$ \\
\hline 231.234692 & -29.413294 & $15245632-2924478$ & -26 & -32 & 0.88 & 10.32 & M4.5-M5 & 116 & $-10: 6$ & $<-2.76$ \\
\hline 232.789928 & -35.082542 & $15310958-3504571$ & -34 & -30 & 0.92 & 10.67 & M4.5-M5 & 70 & $-10: 6$ & $<-3.12$ \\
\hline 233.912086 & -38.210953 & 15353890-3812394 & -30 & -38 & 0.89 & 10.31 & M4-M4.5 & 126 & $-5: 11$ & $<-2.94$ \\
\hline 234.257429 & -36.814156 & $15370178-3648509$ & -16 & -34 & 0.88 & 9.80 & M4-M4.5 & 108 & $-11: 5$ & $<-3.14$ \\
\hline 236.847334 & -36.736374 & $15472336-3644109$ & -26 & -29 & 0.87 & 9.81 & M4.5-M5 & 135 & $-7: 9$ & $<-2.68$ \\
\hline 236.852005 & -36.726772 & $15472448-3643363$ & -24 & -30 & 0.86 & 9.27 & M3-M3.5 & 130 & $-8: 8$ & -3.00 \\
\hline 238.948557 & -36.566711 & $15554765-3634001$ & -30 & -37 & 0.88 & 10.68 & M4-M4.5 & 103 & $-8: 7$ & -2.86 \\
\hline 242.36897 & -32.104786 & $16092855-3206172$ & -12 & -34 & 0.9 & 9.85 & M4.5-M5 & 115 & $-13: 2$ & $<-2.79$ \\
\hline 242.478602 & -30.982832 & $16095486-3058581$ & -18 & -32 & 0.91 & 10.48 & M2-M2.5 & 130 & $-11: 4$ & $<-3.36$ \\
\hline 244.974809 & -31.901608 & $16195395-3154057$ & -24 & -26 & 0.88 & 10.54 & M3-M3.5 & 144 & $-9: 5$ & -2.86 \\
\hline
\end{tabular}

Notes. Spectral types are obtained from photometric colors. RV range is the range of radial velocities that give good $U V W \mathrm{~s}$ (see Section 2.2 for more details). Unless otherwise noted in footnote a, all distances are calculated using $10 \mathrm{Myr}$ isochrones and have uncertainties of 20\%. Upper limits for the last column are derived assuming $F_{\mathrm{X}}=2 \times 10^{-13} \mathrm{erg} \mathrm{cm}^{-2} \mathrm{~s}^{-1}$, the characteristic RASS flux limit (Schmitt et al. 1995).

${ }^{a}$ For these objects distance and RV ranges are quoted using $100 \mathrm{Myr}$ isochrones. 


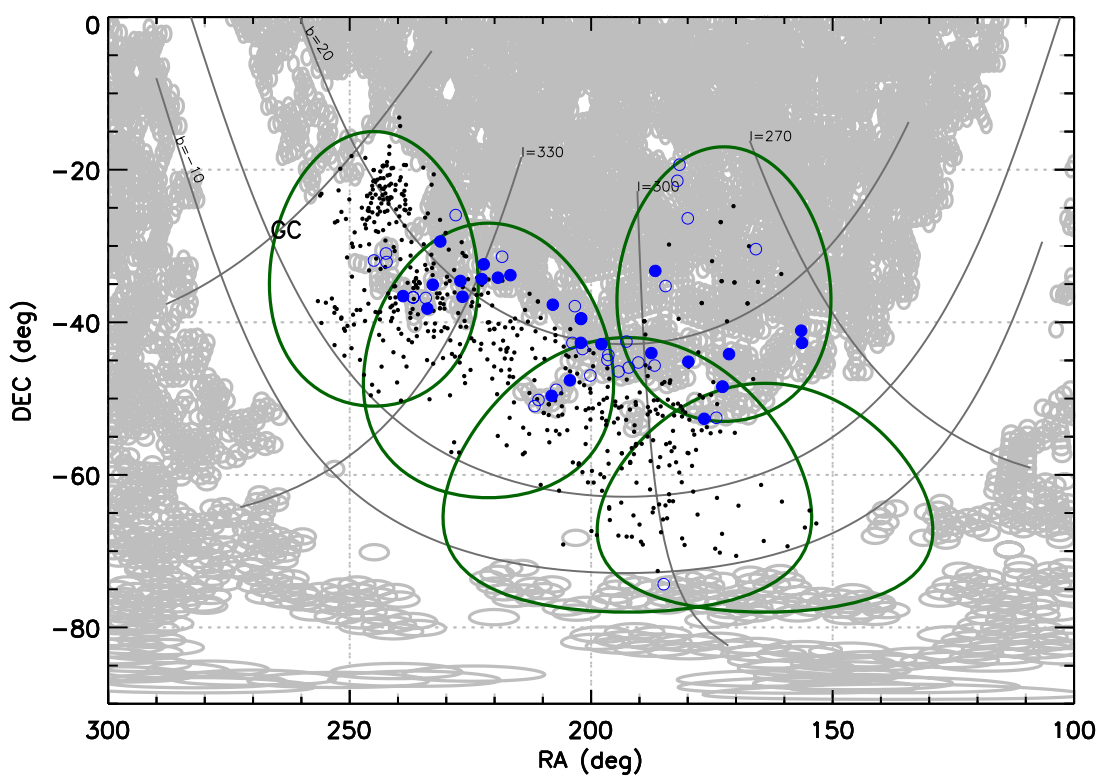

Figure 6. Search regions (see Table 1) for Sco-Cen and TWA. Dots are candidate TWA, US, UCL, and LCC members from Torres et al. (2008) and de Zeeuw et al. (1999). Circles denote candidate objects (Table 2); those we have spectra for are displayed as filled circles (Table 3). Our search regions encompass all known members and candidates for these regions and extend somewhat beyond in order to explore for additional members. All GALEX fields in the GR4/5 database are denoted in gray. Note that GALEX avoids the galactic plane (generally within $10^{\circ}$ ) so that our coverage is better on the northern parts of our searched fields.

(A color version of this figure is available in the online journal.)

Table 3

Spectroscopic Results

\begin{tabular}{ccccccc}
\hline \hline Target & $\begin{array}{c}\text { Spectral } \\
\text { Type }\end{array}$ & $\begin{array}{c}\text { Dist. } \\
(\mathrm{pc})\end{array}$ & $\begin{array}{c}\text { He I } \\
5876 \AA\end{array}$ & $\begin{array}{c}\text { H } \alpha \\
6563 \AA\end{array}$ & $\begin{array}{c}\text { He I } \\
6678 \AA\end{array}$ & $\begin{array}{c}\text { Li I } \\
6708 \AA\end{array}$ \\
\hline $10252092-4241539$ & M1 & 91 & & $-3.6 \pm 0.6$ & & $490 \pm 30$ \\
$10260210-4105537$ & M1 & 67 & & $-7.4 \pm 0.3$ & & $500 \pm 70$ \\
$11254754-4410267$ & M4 & 75 & $-0.8 \pm 0.2$ & $-8.2 \pm 0.2$ & $-50 \pm 30$ & $<30$ \\
$12265135-3316124$ & M5 & 65 & $-6.4 \pm 0.2$ & $-64.0 \pm 4.0$ & $-1200 \pm 200$ & $410 \pm 40$ \\
& & & & $-51.1 \pm 7.0$ & $-670 \pm 110$ & $573 \pm 50$ \\
\hline $11311482-4826279$ & M3 & 130 & & $-7.9 \pm 0.5$ & & $60 \pm 20$ \\
$11462310-5238519$ & M4.5 & 85 & & $-10.1 \pm 0.5$ & & $66 \pm 20$ \\
$11592786-4510192$ & M4.5 & 55 & & $-8.18 \pm 0.3$ & & $527 \pm 50$ \\
$12300521-4402359$ & M4 & 69 & & $-7.2 \pm 0.2$ & & $503 \pm 30$ \\
$13112902-4252418$ & M1.5 & 121 & & $-4.2 \pm 0.2$ & & $255 \pm 20$ \\
$13281440-3929147$ & M4 & 84 & & $-8.7 \pm 0.2$ & & $165 \pm 20$ \\
$13283247-4241436$ & M2 & 111 & & $-3.8 \pm 0.2$ & & $180 \pm 20$ \\
$13373839-4736297$ & M3.5 & 126 & $-1.3 \pm 0.1$ & $-13.7 \pm 0.2$ & $-115 \pm 50$ & $308 \pm 40$ \\
$13514567-3742004$ & M1 & 119 & & $-2.2 \pm 0.1$ & & $200 \pm 10$ \\
$13524567-4938327$ & M4.5 & 112 & & $-8.5 \pm 0.1$ & & $517 \pm 50$ \\
$14265175-3349356$ & M3.5 & 139 & $-0.6 \pm 0.1$ & $-10.2 \pm 0.4$ & $-24 \pm 10$ & $<50$ \\
$14371322-3409209$ & M3.5 & 145 & & $-9.7 \pm 0.6$ & & $<30$ \\
$14485880-3224083$ & M4.5 & 132 & & $-11.8 \pm 0.4$ & & $<60$ \\
$14503359-3420305$ & M3.5 & 142 & $-1.0 \pm 0.1$ & $-10.5 \pm 0.4$ & $<-30$ & $<80$ \\
& & & & $-6.9 \pm 0.6$ & & $<50$ \\
$15063505-3639297$ & M5 & 118 & $-1.9 \pm 0.3$ & $-12.1 \pm 0.3$ & $-44 \pm 20$ & $446 \pm 90$ \\
$15081653-3434466$ & M3.5 & 140 & & $-7.8 \pm 0.5$ & & $<60$ \\
$15245632-2924478$ & M4.5 & 116 & & $-8.7 \pm 0.6$ & & $<100$ \\
$15310958-3504571$ & M4.5 & 70 & $-2.0 \pm 0.2$ & $-17.4 \pm 0.3$ & $-145 \pm 60$ & $258 \pm 40$ \\
$15353890-3812394$ & M4 & 126 & & $-5.97 \pm 0.3$ & & $388 \pm 40$ \\
$15554765-3634001$ & M3.5 & 103 & $-1.0 \pm 0.2$ & $-13.6 \pm 0.2$ & $-39 \pm 10$ & $184 \pm 20$ \\
\hline
\end{tabular}

Notes. The first four objects are from the TWA search, the rest are from the Sco-Cen searches (see Table 1). Some candidate objects found in the Sco-Cen searches may be TWA candidates (see Figure 6). Spectral types are estimated using the TiO5 index as described in Reid et al. (1995) and distances assume the star is $\sim 10$ Myr old. He I (5876 ̊) and H $\alpha$ EWs are measured in $\AA$, all others are in mÅ, with negative EWs denoting emission lines. Upper limits $(3 \sigma)$ are provided when a line is not detected. The lower rows in the entries for 2M1226-33 and 2M1450-34 give equivalent widths measured from the R7000 spectra. 


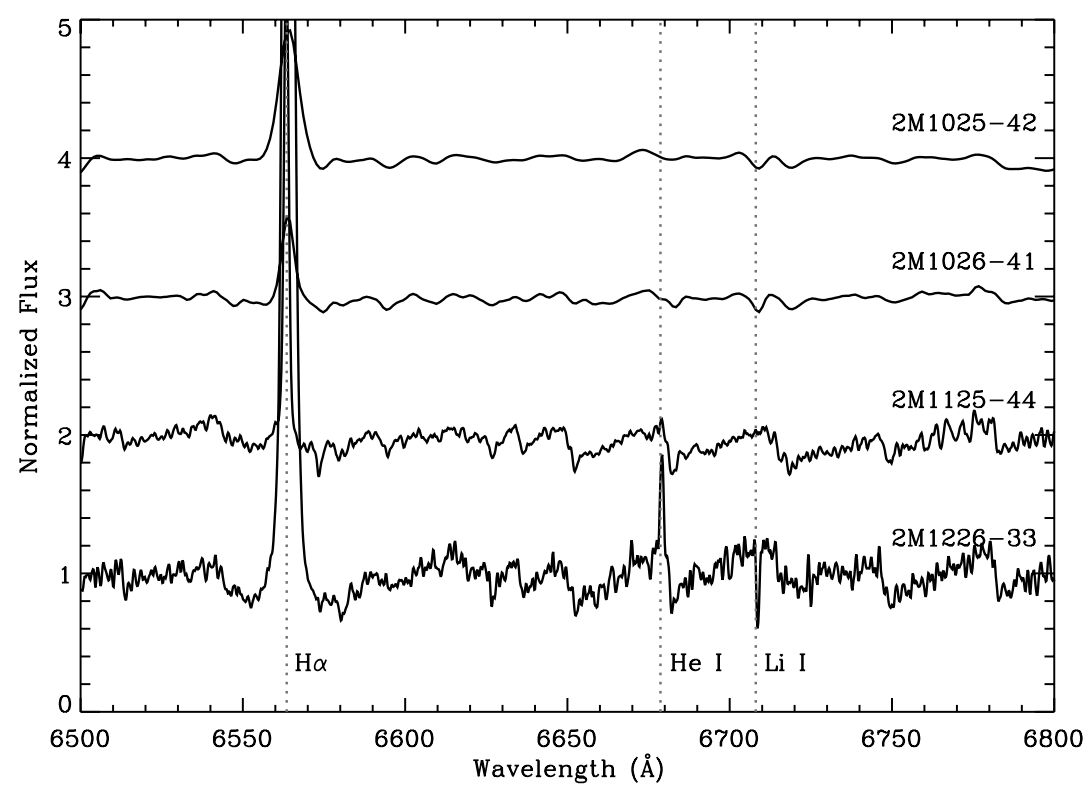

Figure 7. $R_{3000} \mathrm{WiFeS}$ spectra for our four TWA candidates. The spectra for $2 \mathrm{M} 1125-44$ and $2 \mathrm{M} 1226-33$ were obtained with $R=7000$.

\subsection{Spectroscopic Follow Up}

To check for signatures of youth, such as the presence of lithium absorption at $6708 \AA$, we obtained $R=3000$ spectra of the 24 stars listed in Table 3 with the Wide Field Spectrograph (WiFeS) at the Siding Springs Observatory's $2.3 \mathrm{~m}$ telescope during 2010 March and 2010 May. We also acquired $R=7000$ spectra for 5 of our 24 objects in 2010 August. Four of these targets were selected in our TWA search and 20 in our Sco-Cen searches, though a few Sco-Cen candidates overlap with the TWA search region (see Figure 6). WiFeS (Dopita et al. 2007) is a double-beam, image-slicing integral field spectrograph, and provides a $25 \times 38$ arcsec field with 0 '.5 pixels. A variety of gratings can be used to provide $R=3000$ and 7000 spectra. We used the $B_{3000}$ and $R_{3000}$ gratings to cover the wavelength range from 3200 to $9800 \AA$. The spectra for the region covering $\mathrm{H} \alpha$ and $\mathrm{Li} 6708 \AA$ are displayed in Figures 7 and 8. We have measured equivalent widths (EWs) of $\mathrm{H} \alpha, \mathrm{He}$ I, and $\mathrm{Li}$ I for these objects (when detected) and summarize these values in Table 3. Spectral types listed in Table 3 are estimated using the TiO5 index as described in Reid et al. (1995), with an estimated precision of \pm 0.5 in subclass.

\subsection{Results and Discussion}

Of the 24 targets listed in Table 3, 17 display evidence of $\mathrm{Li}$ absorption at $6708 \AA$. The remaining seven have no Li or an unidentified weak/moderate feature is present at a slightly bluer wavelength (offset by $2-3 \AA$ ). Those objects with the weak feature near $\mathrm{Li}$ were re-observed with $R=7000$ (see below). Note, however, that all systems with no evidence of $\mathrm{Li}$ in their spectra have spectral types M3.5-M4.5. As previously mentioned, stars of these spectral types will burn lithium in as little as $\sim 10 \mathrm{Myr}$ and so a lack of lithium does not immediately imply the system is old. Of those that do display some lithium absorption, six are M1-M3, with the remaining being later than M3.5. Those later than M3.5 must be at most $\sim 10$ Myr old in order to show such strong lithium absorption. The earlier M-types will burn their lithium somewhat more slowly, so these systems may be of age $\sim 10$ Myr.
X-ray detection has been one way young stars have been identified (e.g.,, Kastner et al. 1997; Song et al. 2003; Torres et al. 2006). Only 5 of our 24 spectroscopic targets (and only 14 among the 54 candidates) have ROSAT All-Sky Survey (RASS) $\mathrm{X}$-ray detections within $1^{\prime}$ of the target's 2MASS coordinates in either the Bright or Faint source catalogs. The lack of X-ray detection in RASS suggests these objects, if they are young, are producing too little $\mathrm{X}$-ray emission to be readily detected at these distances. Table 2 lists $L_{\mathrm{X}} / L_{\text {bol }}$ for all candidates, including upper limits for RASS non-detections (assuming an RASS flux limit of $2 \times 10^{-13} \mathrm{erg} \mathrm{cm}^{-2} \mathrm{~s}^{-1}$; Schmitt et al. 1995). All RASSdetected candidates have $\log L_{\mathrm{X}} / L_{\mathrm{bol}} \sim-3$, which is typical of nearby M-stars (see Figure 5 in Riaz et al. 2006).

$\mathrm{H} \alpha \mathrm{EW}$ is one way that T Tauri stars are identified and classified. For these spectral types, values for $\mathrm{H} \alpha \mathrm{EW} \geqslant 10-20 \AA$ are typically observed in classical T Tauri stars (White \& Basri 2003). Of the stars observed spectroscopically, nine show $\mathrm{H} \alpha$ EW larger than $10 \AA$, with a 10th system having an $\mathrm{H} \alpha$ EW consistent with $10 \AA$. In addition to $\mathrm{H} \alpha$, we also measure He I EW at 5876 and $6678 \AA$ in those systems with clear He I emission. The eight systems with measured He I also have strong $\mathrm{H} \alpha$. Of these, three have no lithium absorption detected, but have spectral types M3.5-M4. The detected He I and lack of Li may suggest these systems are young, but somewhat older than $\sim 10$ Myr. We comment on several noteworthy targets below for which we acquired $R=7000$ spectra.

2M1125-44: This is an M4 star detected in our TWA search, with a photometric distance of $75 \mathrm{pc}$ if $10 \mathrm{Myr}$ old. The $R=3000$ spectrum suggested a dip near $6708 \AA$, but a higher resolution spectrum revealed no detectable $\mathrm{Li}$, though $\mathrm{He}$ I emission lines at 5876 and $6678 \AA$ are detected. The radial velocity for this system $\left(19.5 \pm 2 \mathrm{~km} \mathrm{~s}^{-1}\right.$; S. Murphy 2010, private communication), combined with the $10 \mathrm{Myr}$ old photometric distance estimate, provides $U V W$ that are consistent with those of young stars. At a distance of $75 \mathrm{pc}$, the Galactic $U V W$ are $-4 \pm 5,-23 \pm 3$, $-4 \pm 5 \mathrm{~km} \mathrm{~s}^{-1}$. The lack of $\mathrm{Li}$, however, implies that the star is older than $\sim 10 \mathrm{Myr}$, but even at $\sim 30 \mathrm{Myr}$ (implying a closer distance of about $50 \mathrm{pc}$ ) the $U V W$ are still consistent with those of a young star $\left(-1 \pm 4,-22 \pm 2,-1 \pm 4 \mathrm{~km} \mathrm{~s}^{-1}\right)$. This star 

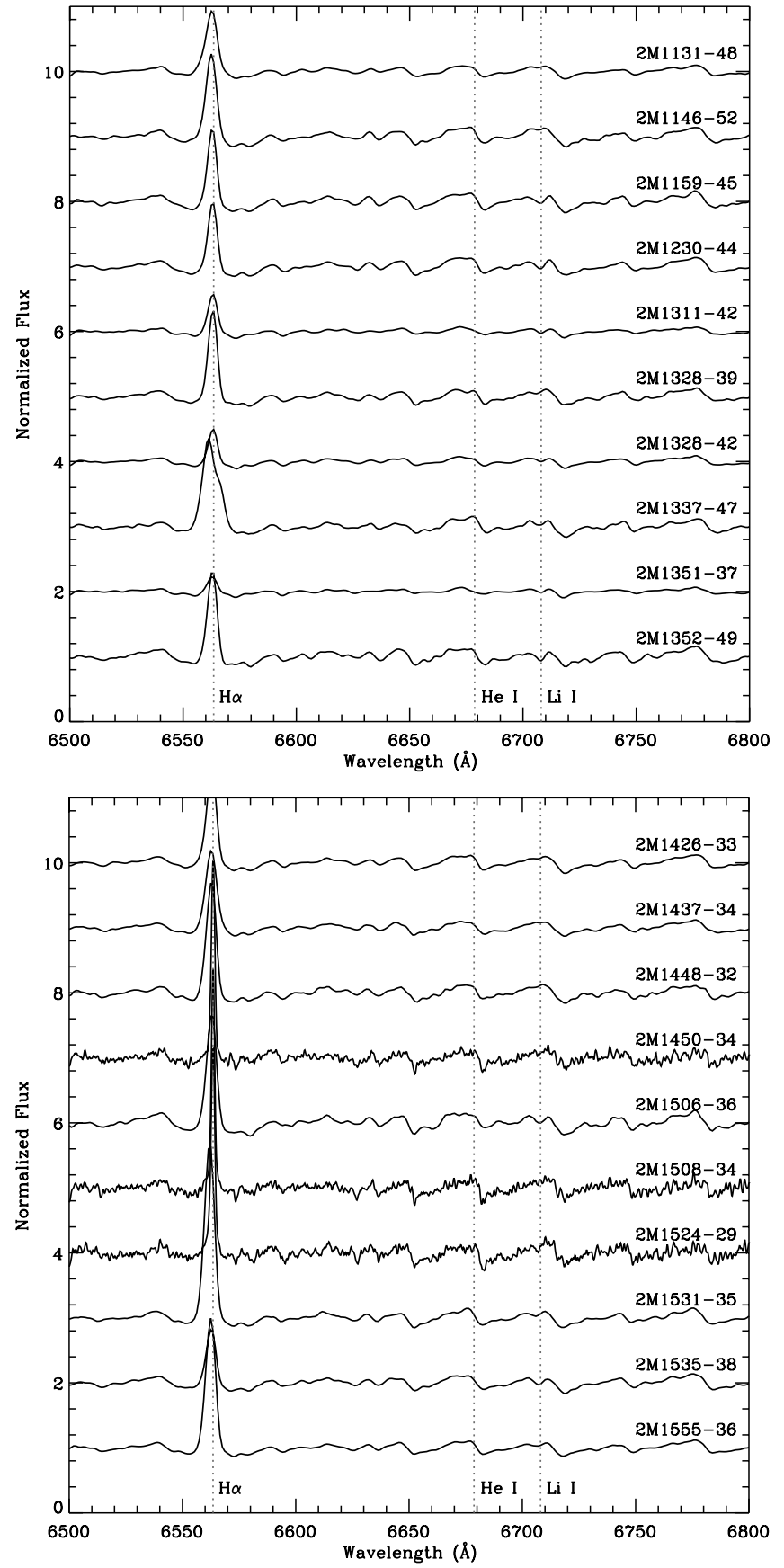

Figure 8. WiFeS spectra for our 20 Sco-Cen candidates. 2M1337-47, which shows asymmetry in its (blueshifted) $\mathrm{H} \alpha$ profile, also shows a double peak for the other hydrogen lines possibly due to accretion from a surrounding disk (Kurosawa et al. 2006). The spectra for 2M1450-34, 2M1508-34, and 2M1524-29 were obtained with $R=7000$.

has been detected in X-rays by the ROSAT All Sky Survey and has $\log L_{\mathrm{X}} / L_{\mathrm{bol}} \sim-2.33$. Hence, this candidate is possibly a young star based on dynamics and X-ray emission, though is not likely to be a member of the $\sim 8 \mathrm{Myr}$ TWA. The $U V W$ is marginally consistent with that of TWA $\left(-11,-18,-5 \mathrm{~km} \mathrm{~s}^{-1}\right.$; Zuckerman \& Song 2004) and the Sco-Cen region (UCL: -6.8, $-19.3,-5.7$, LCC: $-8.2,-18.6,-6.4$, with dispersions of 3-7 $\mathrm{km} \mathrm{s}^{-1}$; Sartori et al. 2003). A parallax measurement will be key in determining the age of this object and membership in any nearby association.
2M1226-33: This is an M5 star detected in our TWA search with a photometric distance of $65 \mathrm{pc}$ if $10 \mathrm{Myr}$ old. This system has very strong $\mathrm{H} \alpha$ and $\mathrm{He}$ lines. The $\mathrm{H} \alpha$ has EW of $64 \pm 4 \AA$ and the 5876 and $6678 \AA$ He I lines have EWs $\sim 6$ and $\sim 1 \AA$, respectively. Strong lithium absorption is also observed (410 $\mathrm{m} \AA$ ). The presence of such strong lines suggests the system may be a classical T Tauri star (White \& Basri 2003). We obtained an additional spectrum for this star using $R=7000$. The EWs differ somewhat from the $R=3000$ spectrum and are listed as an additional row in Table 3. White \& Basri (2003) find that $\mathrm{H} \alpha$ full widths at $10 \%$ higher than $270 \mathrm{~km} \mathrm{~s}^{-1}$ are found among classical $\mathrm{T}$ Tauri stars. With the higher resolution spectrum, we measured a full width at the $10 \%$ level for $\mathrm{H} \alpha$ of $300 \mathrm{~km} \mathrm{~s}^{-1}$, suggesting this object could be a nearby classical T Tauri system. Comparison with Figure 3 in Natta et al. (2004) suggests an accretion rate of $\sim 10^{-10} M_{\odot} \mathrm{yr}^{-1}$. With the higher resolution spectrum, we were also able to determine a radial velocity of $14.8 \pm 3 \mathrm{~km} \mathrm{~s}^{-1}$. We calculate $U V W$ of $-8 \pm 3,-24 \pm 4$, $-1 \pm 3 \mathrm{~km} \mathrm{~s}^{-1}$. The $U V W$ is marginally consistent with that of TWA and Sco-Cen. There is no RASS detection for this object, implying $\log L_{\mathrm{X}} / L_{\mathrm{bol}}<-3.10$; the field has not been observed with Chandra or XMM.

2M1450-34, 2M1508-34, and 2M1524-29: The $R=3000$ spectra for these three stars had an absorption feature near lithium, but offset by 2-3 $\AA$ from the expected $6708 \AA$. A higher resolution spectrum reveals no clear signature of lithium, though a blended $\sim 100 \mathrm{~m} \AA$ feature is seen in 2M1524-29 at a wavelength of $6707.1 \AA$ (see Figure 8). 2M1450-34's $R=3000$ spectrum shows possible He I emission at $6678 \AA$, but this is not seen in the $R=7000$ spectrum (the $R=7000$ spectrum does not cover the region where He I $5876 \AA$ can be seen). The radial velocities measured from the $R=7000$ spectrum are 7.3, 4.9, and $6.0 \mathrm{~km} \mathrm{~s}^{-1}$ (all with uncertainties of $\pm 3 \mathrm{~km} \mathrm{~s}^{-1}$ ) for $2 \mathrm{M} 1450-34$, 2M1508-34, and 2M1524-29, respectively. With 10 Myr photometric distance estimates, the $U V W$ for 2M1450-34 are: $-5 \pm 3,-29 \pm 6,-6 \pm 3 \mathrm{~km} \mathrm{~s}^{-1}$; for $2 \mathrm{M} 1508-34:-7 \pm 3$, $-29 \pm 6,-5 \pm 3 \mathrm{~km} \mathrm{~s}^{-1}$; and for 2M1524-29: $-1 \pm 3,-23 \pm 5$, $-3 \pm 3 \mathrm{~km} \mathrm{~s}^{-1}$. Both $2 \mathrm{M} 1450-34$ and $2 \mathrm{M} 1508-34$ have $U V W$ that appear to be marginally consistent with those of TWA and Sco-Cen. None of these objects have detections in RASS (note that all three stars have photometric distances larger than $100 \mathrm{pc}$; see Table 2), nor have they been observed by Chandra or XMM.

\subsection{Two Stars with Unusual NUV-J Color}

While carrying out the search described in Sections 3.1-3.3, we found two systems in Torres et al. (2008) with particularly unusual NUV- $V$ and NUV- $J$ colors (Figures 2 and 4).

BD +01 2447: This M2 star is listed in Torres et al. (2008) as an AB Dor Association member (age 70 Myr). BD +01 2447, also known as HIP 51317, is 7 pc away, with an upper limit of $60 \mathrm{~m} \AA$ for Li EW (Zickgraf et al. 2005). For an M2 star, however, lithium may be depleted within $\sim 12$ Myr (see Figure 3 in Zuckerman \& Song 2004). The kinematics of the system are consistent with membership in the AB Dor Association and X-ray emission has been detected $\left(\log f_{X} / f_{V} \sim-3.53\right.$; Zickgraf et al. 2005). This object has a 2 MASS $K$ magnitude of 5.311 , which (at $7 \mathrm{pc}$ ) corresponds to $M_{K}$ of 6.1 . The models of Baraffe et al. (1998) predict an M2 star would reach such an $M_{K}$ in about 80-100 Myr. These results are consistent with this being a nearby AB Dor member. However, in Figures 1, 2, and 4 , this system is located in the region of color-color (and color-magnitude) space more common for older systems. It is 
noteworthy that, of all of the $\leqslant 70 \mathrm{Myr}$ stars in Torres et al. (2008) detected by GALEX, BD +01 2447 is the only star (excepting TW Hya, see below) that lies significantly outside the locus of young stars. BD +01 2447 also displays slow rotation ( $v \sin i<3 \mathrm{~km} \mathrm{~s}^{-1}$ ), which is more commonly seen in older systems (T. Forveille 2010, private communication). A closer examination of BD +01 2447 is warranted so as to understand its weak NUV emission.

TW Hya: This star, namesake of the TWA, stands out in all our NUV figures due to its substantial NUV excess. In particular, it has NUV- $J \sim 6$, while the rest of the TWA stars have NUV- $J$ $\sim 9-11$. If the UV excesses displayed by the TWA stars can be ascribed to chromospheric activity due to their youth, an additional emission source is required to explain why TW Hya is $\sim 3$ mag brighter in NUV than the rest of the TWA. Of the young stars plotted in our figures, TW Hya is the only known classical $\mathrm{T}$ Tauri star. The most likely explanation for the increased UV emission is ongoing accretion onto the star, as suggested by its optical and X-ray emission properties $\left(\sim 10^{-8} M_{\odot} \mathrm{yr}^{-1}\right.$; Kastner et al. 2002). Additionally, the orientation of the system is such that we are seeing its accretion unobscured (the disk is near face-on; Krist et al. 2000). Our unobscured view of the accretion onto this star is therefore the likely explanation for its high UV flux. This suggests that, barring intervening absorbing material, actively accreting stars such as TW Hya will readily stand out in $G A L E X$-Optical/NIR colors.

\section{CONCLUSIONS}

We have demonstrated a new technique based on GALEX and 2MASS colors that can readily identify young, low-mass stars near the Earth. Findeisen \& Hillenbrand (2010) similarly demonstrated that such a UV/NIR search strategy is feasible for stars younger than $\sim 5 \mathrm{Myr}$, but essentially no such stars are known to exist within $\sim 100$ pc of Earth. The present study demonstrates that GALEX NUV fluxes may be used in conjunction with either optical or NIR fluxes to identify nearby stars with ages in the range 10-100 Myr and spectral types K5 or later. With additional information, namely space velocities, we have formulated an efficient method to identify candidate members of nearby stellar associations.

We have performed a search in the TWA and Sco-Cen region and recovered 54 high-quality candidates. Of these, we observed 24 spectroscopically and detected lithium absorption, an indicator of youth, in at least 17 M1-M5 systems. Those with no lithium absorption have spectral types M3.5-M4.5, a range where lithium is believed to be depleted within $\sim 10 \mathrm{Myr}$ (Song et al. 2002; Zuckerman \& Song 2004; Yee \& Jensen 2010). These systems may very well be young, albeit not younger than 10 Myr. Detection of He I emission at 5876 and $6678 \AA$ for eight systems (some of which had no lithium absorption) is also consistent with young ages for these stars.

The detection of lithium in at least $2 / 3$ of the 24 spectroscopically observed stars suggests many of the other high-quality candidates may also be young stars, and perhaps others lie among the 100+ UV-excess stars with possible good $U V W$ from which those candidates were drawn. Most of our objects lack RASS detections within $1^{\prime}$ of their 2MASS coordinates; however, these tend to have upper limits of $\log L_{\mathrm{X}} / L_{\text {bol }} \sim-3$, consistent with late-type stars that have been detected in the RASS. Because mid to late M-type stars have small $L_{\text {bol }}$, many would escape detection in RASS even if $\log L_{\mathrm{X}} / L_{\text {bol }} \sim-3$. While deeper $\mathrm{X}$-ray observations can be performed with Chandra or XMM, these will eventually cover only a few percent of the sky. This suggests that UV-selection criteria, such as the one we describe in this paper, may ultimately prove to be a more powerful tool than X-rays to identify nearby, young, low-mass stars.

We thank Simon Murphy for providing the high-resolution spectrum for 2M1125-44 and the referee for useful suggestions. This publication makes use of data products from GALEX, operated for NASA by the California Institute of Technology, and the 2MASS, which is a joint project of the University of Massachusetts and the Infrared Processing and Analysis Center/ California Institute of Technology, funded by the National Aeronautics and Space Administration and the National Science Foundation. This work has used the SIMBAD and VizieR databases, operated at CDS, Strasbourg, France. This research was supported by NASA Astrophysics Data Analysis Program grant NNX09AC96G to RIT and UCLA.

Note added in proof: Shkolnik et al. (2010) performed a similar search in the TWA region and confirm 2M1226-33 as a TWA member (TWA 32) and 2M1131-48 as a likely LCC member.

\section{REFERENCES}

Baraffe, I., Chabrier, G., Allard, F., \& Hauschildt, P. H. 1998, A\&A, 337, 403 Cardelli, J. A., Clayton, G. C., \& Mathis, J. S. 1989, ApJ, 345, 245

Cutri, R. M., et al. 2003, The IRSA 2MASS All-Sky Point Source Catalog, NASA/IPAC Infrared Science Archive, http://irsa.ipac.caltech.edu/ applications/Gator/

de Zeeuw, P. T., Hoogerwerf, R., de Bruijne, J. H. J., Brown, A. G. A., \& Blaauw, A. 1999, AJ, 117,354

Dommanget, J., \& Nys, O. 2002, VizieR Online Data Catalog, 1274, 0

Dopita, M., Hart, J., McGregor, P., Oates, P., Bloxham, G., \& Jones, D. 2007, Ap\&SS, 310, 255

Findeisen, K., \& Hillenbrand, L. 2010, AJ, 139, 1338

Gershberg, R. E., Katsova, M. M., Lovkaya, M. N., Terebizh, A. V., \& Shakhovskaya, N. I. 1999, A\&AS, 139, 555

Gliese, W., \& Jahreiß, H. 1991, in The Astronomical Data Center CD-ROM: Selected Astronomical Catalogs, Vol. I, ed. L. E. Brotzmann \& S. E. Gesser (Greenbelt, MD: NASA/Astronomical Data Center, Goddard Space Flight Center),

Guinan, E. F., \& Engle, S. G. 2009, in IAU Symp. 258, The Ages of Stars, ed. E. E. Mamajek, D. R. Soderblom, \& R. F. G. Wyse (Cambridge: Cambridge Univ. Press), 395

Kastner, J. H., Huenemoerder, D. P., Schulz, N. S., Canizares, C. R., \& Weintraub, D. A. 2002, ApJ, 567, 434

Kastner, J. H., Zuckerman, B., Weintraub, D. A., \& Forveille, T. 1997, Science, 277,67

Krist, J. E., Stapelfeldt, K. R., Ménard, F., Padgett, D. L., \& Burrows, C. J. 2000, ApJ, 538, 793

Kurosawa, R., Harries, T. J., \& Symington, N. H. 2006, MNRAS, 370, 580

Mamajek, E. E., \& Hillenbrand, L. A. 2008, ApJ, 687, 1264

Martin, D. C., et al. 2005, ApJ, 619, L1

Monet, D. G., et al. 2003, AJ, 125, 984

Morrissey, P., et al. 2007, ApJS, 173, 682

Natta, A., Testi, L., Muzerolle, J., Randich, S., Comerón, F., \& Persi, P. 2004, A\&A, 424, 603

Pourbaix, D., et al. 2004, A\&A, 424, 727

Preibisch, T., \& Feigelson, E. D. 2005, ApJS, 160, 390

Preibisch, T., \& Mamajek, E. 2008, in Handbook of Star Forming Regions, Vol. II: The Southern Sky, ed. Bo Reipurth (San Francisco, CA: ASP), 235 Reid, I. N., Hawley, S. L., \& Gizis, J. E. 1995, AJ, 110, 1838

Riaz, B., Gizis, J. E., \& Harvin, J. 2006, AJ, 132, 866

Roeser, S., Demleitner, M., \& Schilbach, E. 2010, AJ, 139, 2440

Röser, S., Schilbach, E., Schwan, H., Kharchenko, N. V., Piskunov, A. E., \& Scholz, R.-D. 2008, A\&A, 488, 401

Sartori, M. J., Lépine, J. R. D., \& Dias, W. S. 2003, A\&A, 404, 913

Schmitt, J. H. M. M., Fleming, T. A., \& Giampapa, M. S. 1995, ApJ, 450, 392

Shkolnik, E. L., et al. 2011, ApJ, 727, 6

Song, I., Bessell, M. S., \& Zuckerman, B. 2002, ApJ, 581, L43

Song, I., Zuckerman, B., \& Bessell, M. S. 2003, ApJ, 599, 342 
Stauffer, J., et al. 2010, PASP, 122, 885

The DENIS Consortium. 2005, VizieR Online Data Catalog, 2263, 0

Torres, C. A. O., Quast, G. R., da Silva, L., de La Reza, R., Melo, C. H. F., \& Sterzik, M. 2006, A\&A, 460, 695

Torres, C. A. O., Quast, G. R., Melo, C. H. F., \& Sterzik, M. F. 2008, in Handbook of Star Forming Regions, Vol. II: The Southern Sky, ed. Bo Reipurth (San Francisco, CA: ASP), 757
Walkowicz, L. M., \& Hawley, S. L. 2009, AJ, 137, 3297

White, R. J., \& Basri, G. 2003, ApJ, 582, 1109

Yee, J. C., \& Jensen, E. L. N. 2010, ApJ, 711, 303

Zacharias, N., et al. 2010, AJ, 139, 2184

Zickgraf, F.-J., Krautter, J., Reffert, S., Alcalá, J. M., Mujica, R., Covino, E., \& Sterzik, M. F. 2005, A\&A, 433, 151

Zuckerman, B., \& Song, I. 2004, ARA\&A, 42, 685 